\title{
ARDUINO BASED SMART IOT FOOD QUALITY MONITORING
}

\author{
Kaviya \\ Department of ECE \\ Saveetha School of engineering, Chennai, Tamil Nadu, India
}

\begin{abstract}
The growing interest in food quality and safety needs the event of sensitive and reliable ways of research yet as technology for freshness preservation and food quality. This review describes the standing of chemical and biological sensors for food observation and good packaging. Sensing styles and their analytical options for measure freshness markers, allergens, pathogens, adulterants and toxicants square measure mentioned with example of applications. Their potential implementation in good packaging might facilitate foodstatus observation, scale back scraps, extend shelf-life, and improve overall food quality. However, most sensors square measure still within the development stage and want important work before implementation in realworld applications. problems like sensitivity, property, robustness, and safety of the sensing materials because of potential contact or migration in food ought to be established. The present development standing of those technologies, beside a discussion of the challenges and opportunities for future analysis, square measure mentioned.
\end{abstract}

Keywords - Food packaging, Hygiene, IoT, Arduino UNO, Wireless network.

\section{INTRODUCTION}

Food safety and hygiene is a major concern in order to prevent the food wastage. The Quality of the food needs to be monitored and it must be prevented from rotting and decaying by the atmospheric factors like temperature, humidity and dark. Therefore, it is useful to deploy quality monitoring devices at food stores. These quality monitoring devices keep a watch on the environmental factor that cause or pace up decay of the food. Later, the environmental factors can be controlled like by refrigeration, vacuum storage etc. Several changes can take place in packaged food as a result of metabolism or microbial growth over time(2019).

It is normal that by 2020, the Internet of Things (IoT) will have become quicker than some other classification of associated devices, according to. Also, about 34 billion USD will be spent on IoT gadgets, as per a similar report. On the worldwide level, administrations offered by the IoT and Machine-to-Machine(2011).
Correspondence (M2M) associations will increment by very nearly multiple times, from 4.9 billion out of 2015 to 12.2 billion until 2020 , speaking to practically half (46\%) of the complete number of associated gadgets. IoT restorative administrations will increment by five occasions, indicating the quickest advancement, from 144 million associations in 2015 , to 729 million of every 2020 , as per the examinations. The IoT is likewise expected to make a significant commitment in the related fields of nourishment and customer security. The electronic gas sensor, a common application in insightful and IoT systems, is getting ceaselessly expanding consideration from both the mechanical and the scholarly condition. Gas identification innovation is quickly advancing a result of its wide scope of utilizations. Gas sensors can be ordered by the distinguishing materials utilized in their creation procedure, by their electrical parameters, by the recognized gases, or by their expenses. Portable olfaction and smell source limitation, innovations with high effect on insightful nourishment highlight extraction, have become significant fields of research as of late. Late advancement in versatile mechanical autonomy and the dynamic idea of the working conditions the robots are working in have expanded the preparing intensity of the robots. Moreover, a few cartographic methods have been produced for individual or different robots equipped for restricting smell sources. At last, a basic methodology dependent on a conduct model has been executed on a robot fitted with electronic smell and sight in powerful conditions so as to maintain a strategic distance from the high time utilization and inflexibility of the calculation based methodology. A remote electronic nose (WEN) produced for natural product quality control and characterization was demonstrated in. The electronic nose system was based on wireless data transmission using ZigBee, a series of gas sensors and a LabVIEW Graphic User Interface, and it was tried with green and yellow mango tests. The joining of sensors in nourishment bundling gives the buyer keen bundling arrangements. These advances have prompted improved nourishment quality, wellbeing, longer timeframe of realistic usability and convenience. While most bundling advancements have been the after effect of worldwide patterns and purchaser inclinations, a couple of developments have originated from rather sudden sources, for example, the rise of nano-sensors and the innovation of material detecting in $\mathrm{nm}$ size range. Without a doubt, future keen bundling advancements will concentrate on sanitation 
(recognizing microbial development, oxidation, and improved alter perceivability), nourishment quality (detecting unpredictable flavours and smells), timeframe of realistic usability following, validation, accommodation, and manageability of nourishment items. It is demonstrating critical to create gadgets furnished with minimal effort business sensors equipped for distinguishing and estimating the centralizations of the environmental gases. CO2 and methane (CH4), because of their nursery impacts and the potential gas spillages in water wells, can seriously jeopardize human and creature wellbeing. The framework we propose is expected for nourishment quality checking. It constantly screens the gas level, the mugginess level and the temperature of vacuum-pressed nourishments.

\section{LITERATURE SURVEY}

In recent years, computing systems have shown a rapid growth, with their explosive evolution benefiting almost all sectors of human activity. Nowadays, mobility has become a highly prized quality. As "smartphone"-type mobile phones are becoming more and more affordable in terms of price, quality and performance, applications developed on the Android platform have the potential to become extremely useful in conjunction with database management systems[11].

The internet of things (IoT) is a concept and, implicitly, a model built on the assumption of the omnipresence of a multitude of objects which, through wireless or cabled connections and unique addressing schemes, can interact and collaborate with other objects, creating new services and applications aimed at reaching a common goal [15]. In this light, the promise of an intelligent environment is extremely seductive, envisioning a world where reality and the artificial digital virtual components of the world interact in order to create a better environment. Being a new Internet revolution, IoT's goal is to allow things and objects to be connected anytime and anywhere with anyone, using any network, path and service. Because devices can provide information about themselves, objects are recognized and learned by making contextual decisions. They can access information that has been used in a connection with other things, or they can be parts of complex services.

The future trend in intelligent packaging is a global expansion in food distribution systems. The quality and safety of vacuum foods are strongly influenced by temperature, making the monitoring of time- temperature history throughout the entire distribution chain compulsory. Commercially available monitoring devices include temperature data loggers, time-temperature integrators (TTIs), smart radio frequency identification (smart-RFID), and others.

\section{FRESHNESS INDICATOR FOR DIFFERENT TYPES OF FOODS:}

- Fish, meat or poultry:

It undergo degradation, different spoilage indicators can be found indicating lipid decay, protein breakdown and ATP decay.

\section{- Fruits and vegetables:}

Technology for monitoring is necessary to decrease the loss during transportation and storage. It produces ethylene during environmental stress which leads to ripening even at extremely low concentration.

\section{- Biosensor for food allergen detection:}

The presence of allergens in food products such as milk, soya bean, eggs, cereals and nuts is an increased safety concern.

\section{PROPOSED SYSTEM}

The nourishment quality checking gadget planned in this venture depends on Arduino UNO. So purchaser ought to know about how to begin with Arduino. The arduino has filled in as an IoT board in this one. Different sensors like DHT-11, MQ3 and LDR, the ESPS266 Wi-Fi modem anad character LCD are interfaced to the arduino.

TABLE -1 PINS IN ARDUINO UNO:

\begin{tabular}{|l|l|l|}
\hline 21 & AREF & Reference Voltage \\
\hline 22 & GND & Ground \\
\hline 23 & PCO & Analog Input \\
\hline 24 & PC1 & Analog Input \\
\hline 25 & PC2 & Analog Input \\
\hline 26 & PC3 & Analog Input \\
\hline 27 & PC4 & Analog Input \\
\hline 28 & PC5 & Analog Input \\
\hline
\end{tabular}

In this venture, the two Analog Input pins of the board are utilized to interface the LDR and MQ3 sensor, one GPIO is utilized to interface DHT-11 sensor, two GPIO are utilized to interface ESP8266 module where pins are designed UART transmitter and recipient pins utilizing programming sequential and 6 GPIO pins are utilized to interface the realistic LCD.

DHT-11 Sensor - DHT-11 is a temperature and stickiness sensor. The DHT11 sensor comprises of two fundamental parts - one is Humidity detecting segment and other is NTC temperature sensor (or Thermistor). The Thermistor is really a variable resistor that changes its opposition with change in temperature. The two of them sense the temperature and moistness of territory and give the yield to the IC (which is put on posterior of sensor). The sensor has four pins - VCC, Ground, information Out and NC. The VCC and Ground pins 


\section{International Journal of Engineering Applied Sciences and Technology, 2020 Vol. 4, Issue 12, ISSN No. 2455-2143, Pages 592-597 \\ Published Online April 2020 in IJEAST (http://www.ijeast.com)}

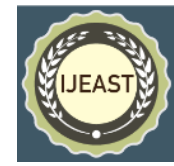

are associated with the regular VCC and Ground individually. The Data Out pin of the sensor is associated with PB0 pin of the Arduino board.

Arduino uno - the arduino uno is atmega328 based microcontroller board. it is one of the most well known prototyping sheets. the board accompanies worked in arduino boot loader. it has 14 gpio pins, 6 pwm pins, 6 analog sources of info and on board uart, spi and twi interfaces, an on-board resonator, a reset catch, and openings for mounting pin headers. while programming the board, it tends to be associated with the pc utilizing usb port and the board can runs on usb power. the arduino uno has $32 \mathrm{~kb}$ flash memory, $1 \mathrm{~kb}$ eeprom and $2 \mathrm{~kb}$ sram. the board can be associated with various arduino shields for availability with ethernet, bluetooth, wi-fi, zigbee or cellular system and it very well may be associated with a large portion of the iot stages.

MQ3 Sensor - MQ3 liquor sensor module is utilized to distinguish the nearness of ethanol, where the delicate material utilized for this sensor is $\mathrm{SnO} 2$, whose conductivity is lower in clean air. It's conductivity increments as the convergence of ethanol gases increments. It has high affectability to liquor and has a decent protection from aggravations because of smoke, fume and fuel. This module gives both computerized and simple yields. It has a high affectability and quick reaction time. Sensor gives a simple resistive yield dependent on liquor focus. MQ-3 is a simple just as computerized sensor. The nearness of ethanol fumes in nourishment is an indication of rot. In this way, by MQ3 sensor, it tends to be distinguished if nourishment has begun rotting. The sensor has four pins Analog Out, Digital Output, VCC and Ground. The VCC and ground are associated with the basic VCC and Ground. The computerized yield pin isn't utilized along these lines is kept not associated. The yield of the sensor is drawn from the simple yield pin which is associated with the pin A0 of the Arduino board.

LDR Sensor - The LDR is utilized to detect the power of light. The sensor is associated with the A1 pin of the Arduino board. The sensor is associated in a potential divider circuit. The LDR gives a simple voltage which is changed over to computerized perusing by the in-fabricated ADC.

16X2 LCD - The 16X2 LCD show is associated with the Arduino board by interfacing its information pins to pins 2 to 5 of the Arduino board. The RS and E pins of the LCD are associated with pins 10 and 9 of the Arduino board separately. The RW pin of the LCD is grounded.

The standard open-source library for interfacing LCD with Arduino board is utilized in the task. The library functions true to form and needs no progressions or adjustments.

ESP8266 Module - The ESP8266 Wi-Fi Module is an independent SOC with coordinated TCP/IP convention stack that can access to a Wi-Fi organize. The ESP8266 is able to do either facilitating an application or off stacking all Wi-Fi organizing capacities from another application processor. Each ESP8266 module comes pre-customized with an AT order set firmware. The module comes accessible in two models - ESP01 and ESP-12. ESP-12 has 16 pins accessible for interfacing while ESP-01 has just 8 pins accessible for use.

The RESET and VCC pins of the module are associated with the $3.3 \mathrm{~V} \mathrm{DC}$ while Ground pin is associated with the shared conviction. The Tx and Rx pins of the module are associated with the 12 and 13 pins of the Arduino UNO.

Force Supply - The circuit works on 5V DC. The AC mains is utilized as the essential wellspring of intensity. The stock from the mains is ventured somewhere around a transformer and corrected by a full-connect rectifier. The amended yield is managed to $5 \mathrm{~V}$ and $12 \mathrm{~V}$ utilizing 7805 and 7812 ICs. The pin 1 of both the voltage controller ICs is associated with the anode of the battery and pin 2 of the two ICs is associated with ground. The separate voltage yields are drawn from pin 3 of the individual voltage controller ICs. A LED alongside a $10 \mathrm{~K} \Omega$ pull-up resistor is additionally associated between shared belief and yield pin to get a visual trace of supply coherence.

\section{WORKING}

This Arduino based IoT gadget ought to be introduced in a nourishment store. When it is appropriately introduced and fueled on, it associates with the web by means of $\mathrm{Wi}-\mathrm{Fi}$ modem and start perusing information from the interfaced sensors - DHT-11 temperature and moistness sensor, MQ3 Sensor and the LDR sensor.

DHT11 Temperature and Humidity Sensor is an advanced sensor with inbuilt capacitive dampness sensor and Thermistor. It transfers a continuous temperature and moistness perusing like clockwork. The sensor works on 3.5 to $5.5 \mathrm{~V}$ supply and can peruse temperature between $0^{\circ} \mathrm{C}$ and $50^{\circ} \mathrm{C}$ and relative stickiness somewhere in the range of $20 \%$ and $95 \%$.

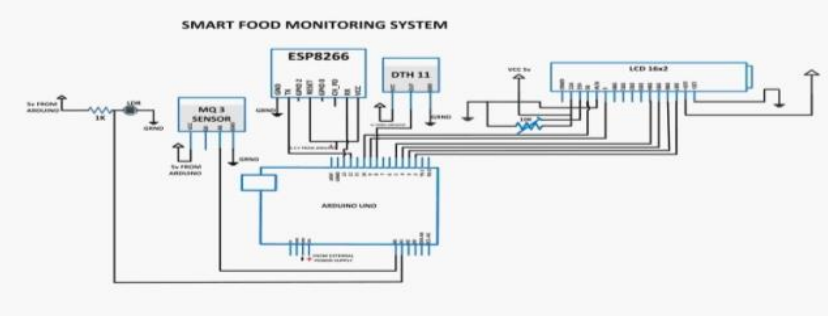

The sensor can't be legitimately interfaced to a computerized pin of the board as it works on 1-wire convention which must be actualized distinctly on the firmware. First the information pin is designed to enter and a beginning sign is sent to it. The beginning sign contains a LOW for 18 milliseconds followed by a HIGH for 20 to 40 microseconds followed by a LOW again for 80 microseconds 


\section{International Journal of Engineering Applied Sciences and Technology, 2020 Vol. 4, Issue 12, ISSN No. 2455-2143, Pages 592-597 Published Online April 2020 in IJEAST (http://www.ijeast.com)}

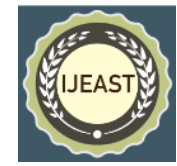

and a HIGH for 80 microseconds. In the wake of sending the beginning sign, the pin is designed to computerized yield and 40-piece information containing the temperature and mugginess perusing is locked out. Of the 5-byte information, the initial two bytes are number and decimal piece of perusing for relative mugginess individually, third and fourth bytes are whole number and decimal piece of perusing for temperature and last one is checksum byte.

For Arduino, standard library for DHT-11 sensor is as of now accessible. The information from the sensor can be effectively prepared by calling read11() technique for the DHT class.

The LDR sensor is associated in a potential divider circuit and information sources a voltage at the simple information pin of the controller. The voltage is perused and digitized utilizing in-constructed ADC channel.

The MQ3 sensor recognizes the discharge of ethanol sort of gases. In the event that the nourishment/organic products get ruined, they transmit the ethanol sort of gases. The MQ3 sensor distinguishes the grouping of such gases and yield a simple voltage corresponding to the convergence of the gas. The simple yield is passed to the simple pin of the Arduino which has inbuilt ADC that coverts the simple to advanced worth.

The Arduino gathers information from every one of the sensors and convert the qualities to the strings. The sensor information wrapped as legitimate strings are passed to the character LCD for show. The ESP8266 Wi-Fi module associated with the Arduino transfers the information to ThingSpeak Server. For showing and checking information transferred to the ThingSpeak server, either an advanced dashboard or information dealer is required. In this task, a computerized dashboard called Freeboard.io is utilized to screen the sensor information outwardly on the web. The Freeboard.io use JASON document to picture ThingSpeak information. It offers three components to assemble a dashboard -

1) Data Sources - The information sources get the information from outer sources. These outer sources can be information specialist administrations, JavaScript applications or JSON records accepting substance from a HTTP server. In this undertaking, the information source is a JSON document that gets information from the ThingSpeak server.

2) Widgets - The Widgets help to show information in literary or graphical structure. There are numerous gadgets accessible in Freeboard.io like content, chart, check and so forth.

3) Panes - These are utilized to sort out gadgets.

\section{ADVANTAGES}

- To remove unwanted compounds

- To prevent micro-organisms proliferation

- To create good conditions for conservation

- To increase the productivity

\section{DISADVANTAGES}

- Execution of IoT prompts loss of occupations for untalented representatives who don't adapt to most recent patterns in equipment and programming field. This can occur in occupations related with stock control and computerized machines, for example, ATM/registration machines.

- The IoT comprises of various innovations whose designs are not quite the same as each other. This makes IoT organize an extremely perplexing framework. Because of this reality, disappointment in the IoT system may prompt more opportunity for rebuilding of the administration to buyers. This may require gifted specialists requiring information on different advancements. Subsequently IoT specialist co-op should pay high to contract and hold such workers.

- As there are various merchant gadgets engaged with the IoT organize, it requires interoperability tests to be directed before propelling the IoT framework for use. This will cause costs on the IoT gadget makers and system specialist organizations.

- It will set aside some effort for IoT framework to turn out to be totally steady as there are new and up and coming models being coordinated in the IoT framework. Basic IoT engineering will help in such manner.

\section{CONCLUSION}

As indicated by the World Health Organization, from a restorative perspective, age is related with numerous infections for which there are no compelling medicines. Notwithstanding, a similar report, demonstrating that the maturing populace is related with the expansion in the commonness of the infections and specific related side effects, shows that all these could be deferred by the appropriation of a sound way of life. In this specific circumstance, our examination adds to expand the personal satisfaction, using insightful sensor systems to caution the matured people when a nourishment terminates, or when certain properties of the nourishment bundling are modified. In this article, we introduced a compact framework for observing vacuum pressed nourishments. The reference parameters estimated in the unfilled compartment were practically consistent. At that point we broke down the conduct of $3 \mathrm{~cm}$ width onion cuts in vacuum during $5 \mathrm{~h}$, at room temperature. We decided to test 


\section{International Journal of Engineering Applied Sciences and Technology, 2020 Vol. 4, Issue 12, ISSN No. 2455-2143, Pages 592-597 \\ Published Online April 2020 in IJEAST (http://www.ijeast.com)}

the onion since it is a typical nourishment fixing and is touchy to capacity conditions. The outcomes demonstrated that the ease MQ5gas sensors were firmly influenced by the onion's emanations. Through vacuum preparing, the onion's breath rate bit by bit diminished because of the decrease of its metabolic exercises and the exhaustion of oxygen in the fenced in area. The most significant compound identified in the onion discharges is liquor. The vacuum pressure in the compartment, after a transient period, settled $(5.48 \mathrm{hPa}$ standard deviation at $600 \mathrm{hPa}$ mean). Dampness at first demonstrated an expansion because of the outflow of water from the plant tissue, trailed by a consistent state $(5.66 \%$ standard deviation at $75 \%$ mean). In the writing, the most contemplated parameter in nourishment bundle checking is the temperature. The exploratory outcomes demonstrated that by keeping up low dampness substance and restricting the wounds that quicken oxidative debasement, the onion cuts dried under vacuum can be kept for a more drawn out timeframe contrasted and ordinary drying. The odds of defilement of the item are limited, in light of the fact that the entire procedure happens in a shut chamber. In future works, extra trial information must be gathered so as to improve the framework execution, and the discovery framework a work in progress will be tried for refrigerated vacuum-stuffed nourishments. This framework will be scaled down and will help old and debilitated people by disturbing them if the nourishment begins breaking down. A possibility for interfacing the cell phone with the checking framework will additionally improve the way toward observing the condition of put away nourishment.

\section{ACKNOWLEDGEMENT}

I am over helmed in all humbleness and gratefulness to acknowledge our depth to all those who have helped me to put these ideas, well above the level of simplicity and into something concrete.

We would like to express our sincere gratitude to my Guide Ms.Nithya for providing their invaluable guidance, comments and suggestions throughout the course of the project. We have taken efforts in this project. However, it would not have been possible without the kind support and help of many individuals and organization Faculties. Whose guidance, encouragement, Suggestion and very constructive criticism have contributed immensely to the evolution of ideas on project. I would like to extend our sincere thanks to all of them.

\section{REFERENCE}

[1] Sofian, M.K.; Oussama, M.E.; Imad, A.A.; Marsha, C.K. "Semiconducting metal oxide based sensors for selective gas pollutant detection. Sensors", mdpi, pp. 81588196(2009).
[2] Wisitsoraat, A.; Tuantranont; Comini, E.; Sberveglieri, G.; Wlodarski, W. "Characterization of n-type and p-type semiconductor gas sensors based on NiOx dopedTiO2 thin films", research gate, vol 517,pp. 2775-2780, (2009).

[3] Emadi, T.A.; Shafai, C.; Freund, M.S.; Thomson, D.J.; Jayasz, D.S.; Whitex, N.D.G. " Development of a polymer-based gas sensor-humidity and CO2 sensitivity. In Proceedings of the 2nd Microsystems and Nanoelectronics" Research Conference (MNRC), Ottawa, ON, Canada; pp. 112-115, 13-14 October 2009.

[4] C. Hagleitner, D. Lange, A. Hierlemann, O. Brand and H. Baltes, "CMOS single-chip gas detection system comprising capacitive, calorimetric and mass-sensitive microsensors", in IEEE Journal of Solid-State Circuits, vol. 37, no. 12, pp. 1867-1878, Dec. 2002.

[5] J. Siden, X. Zeng, T. Unander, A. Koptyug and H. Nilsson, "Remote Moisture Sensing utilizing Ordinary RFID Tags," SENSORS, 2007 IEEE, Atlanta, GA, 2007, pp. 308-311.

[6] T. T. Thai, L. Yang, G. R. DeJean and M. M. Tentzeris, "Nanotechnology Enables Wireless Gas Sensing," in IEEE Microwave Magazine, vol. 12, no. 4, pp. 84-95, June 2011.

[7] Wang, Chengxiang; Yin, Longwei; Zhang, Luyuan; Xiang, Dong; Gao, Rui. 2010. "Metal Oxide Gas Sensors: Sensitivity and Influencing Factors." Sensors 10, no. 3: 2088-2106.

[8] Kuswandi, Bambang \& Wicaksono, Yudi \& Abdullah, Aminah \& yook heng, Lee \& Musa, Ahmad. "2 3 Sensing and Instrumentation for Food Quality and Safety Smart packaging: sensors for monitoring of food quality and safety”. Vol 5. PP 137-146(2011).

[9] P. Sundaravadivel, K. Kesavan, L. Kesavan, S. P. Mohanty and E. Kougianos, "Smart-Log: A DeepLearning Based Automated Nutrition Monitoring System in the IoT," in IEEE Transactions on Consumer Electronics, vol. 64, no. 3, pp. 390-398, Aug. 2018.

[10] Bing Jia and Yongjian Yang, "The design of food quality supervision platform based on the Internet of Things," Proceedings 2011 International Conference on Transportation, Mechanical, and Electrical Engineering (TMEE), Changchun, 2011, pp. 263-266.

[11] S. Bhushan, B. Bohara, P. Kumar and V. Sharma, "A new approach towards IoT by using health care-IoT and food distribution IoT," 2016 2nd International Conference on Advances in Computing, Communication, \& Automation (ICACCA) (Fall), Bareilly, 2016, pp. 1-7.

[12] P. P. Ray, S. Pradhan, R. K. Sharma, A. Rasaily, A. Swaraj and A. Pradhan, "IoT based fruit quality measurement system," 2016 Online International Conference on Green Engineering and Technologies (ICGET), Coimbatore, 2016, pp. 1-5. 
International Journal of Engineering Applied Sciences and Technology, 2020

Vol. 4, Issue 12, ISSN No. 2455-2143, Pages 592-597

Published Online April 2020 in IJEAST (http://www.ijeast.com)

[13] I. Chiuchisan and O. Geman, "Trends in embedded systems for e-Health and biomedical applications, " 2016 International Conference and Exposition on Electrical and Power Engineering (EPE), Iasi, 2016, pp. 304-308.

[14] I. Chiuchisan, H. Costin and O. Geman, "Adopting the Internet of Things technologies in health care systems," 2014 International Conference and Exposition on Electrical and Power Engineering (EPE), Iasi, 2014, pp. 532-535.

[15] Alexandru Popa 1 , Mihaela Hnatiuc 2 , Mirel Paun 2 ,'An Intelligent IoT-Based Food Quality Monitoring Approach Using Low-Cost Sensors”, MDPI , 13 March 2019. 